\title{
THE EFFECT OF COOLING RATE ON STRUCTURE AND MECHANICAL PROPERTIES OF Co-Cr-Cu-Fe-Ni-Sn HIGH ENTROPY ALLOYS
}

\author{
O.I. Kushnerov*, V.F. Bashev \\ Oles Honchar Dnipro National University, Dnipro, Ukraine \\ *e-mail: kushnrv@gmail.com
}

The paper examines the structure and mechanical properties of multicomponent high-entropy CoCrCuFeNiSn $_{\mathrm{x}}$ alloys in as-cast and splat-quenched states. The compositions of investigated alloys is analyzed by using the criteria for predicting the phase composition of high-entropy alloys available in the literature, based both on calculations of the entropy and enthalpy of mixing and on an estimate of the difference between the atomic radii of the component. The alloy films is fabricated by the known technique of splat-quenching. A cooling rate estimated by a film thickness is $\sim 10^{6} \mathrm{~K} / \mathrm{s}$. The simultaneous formation of two solid solutions (FCC + ordered BCC) has been established in the alloy structure. An increase in the concentration of Sn favors the formation of the ordered (B2 type) solid solution in the structure of the alloys. High values of the microhardness and dislocation density have been obtained in the splat-quenched samples. It is also shown that an increase in the Sn content positively affects the microhardness.

Keywords: high entropy alloy, structure, phase composition, splat-quenching, microhardness.

Received 27.06.2021; Received in revised form 29.07.2021; Accepted 15.08.2021

\section{Introduction}

High entropy alloys are one of the most recent and promising discoveries in materials science. Since 2004, significant research attention has been paid to a new class of materials called multi-basic element alloys (MPEA), high entropy alloys (HEA), or complex concentrated alloys (CCA). Such alloys usually contain from 5 to 13 elements in equiatomic or close to equiatomic concentrations [1]. The correct choice of the number of components and their concentrations makes it possible to obtain an alloy with a high mixing entropy, the value of which is preserved both in the melt and after solidification. Simple solid solutions of substitution with face-centered or body-centered cubic crystal lattices are usually formed during the crystallization of such multicomponent alloys because of their high mixing entropy. On the other hand, it is possible to obtain the HEAs with a structure in which intermetallic compounds with high hardness values (Laves phases, $\sigma$ phase) will be combined with a simple solid solution characterized by high ductility [1]. Many high-entropy alloys possess unique properties, such as wear-resistance, resistance to corrosion and oxidation, radiation resistance, high hardness and strength [1-6]. It should also be noted the superior biocompatibility demonstrated by some HEAs [4]. So, the HE alloys may find use as materials for nuclear reactors applications, medicine, electronics devices, mechanical equipment, rocket casings, and engines, etc. Recently the amorphous high-entropy alloys have been successfully synthesized [7-9]. Such alloys were called high entropy metallic glasses (HE-MGs). The development of HE-MGs alloys was the basis for new methods of development and manufacturing of MGs. The HE-MGs (as happens with substances that simultaneously belong to different types of materials $[10,11])$ have excellent physical and mechanical properties combining the advantages of both metallic glasses and high-entropy alloys [12]. Therefore, HE-MGs exhibit considerable potential for various applications.

The application of the liquid quenching method with cooling rates of up to $10^{8} \mathrm{~K} / \mathrm{s}$ allows one to obtain new metastable states in alloys, including highly supersaturated solid solutions and intermediate metastable phases, in particular, a solid amorphous state, with improved physical characteristics [13].

In this work, we investigate the influence of the rapid quenching from melt on the phase composition and the improvement of some physical properties, in particular, the microhardness of multicomponent high-entropy $\mathrm{CoCrCuFeNiSn}_{\mathrm{x}}(\mathrm{x}=0.5 ; 1.0)$ alloys. 


\section{Experimental details}

The as-cast samples of $\mathrm{CoCrCuFeNiSn}$ alloys with nominal compositions presented in Tab.1. were prepared with a Tamman high-temperature electric furnace in the argon gas flow using a copper mold.

Table 1

\begin{tabular}{|l} 
Nominal chemical compositions of CoCrCuFeNiSn \\
\begin{tabular}{|c|c|c|c|c|c|c|}
\hline & $\mathrm{Co}$ & $\mathrm{Cr}$ & $\mathrm{Cu}$ & $\mathrm{Fe}$ & $\mathrm{Ni}$ & $\mathrm{Sn}$ \\
\hline Composition of $\mathrm{CoCrCuFeNiSn}_{0.5}$, at.\% & 18.18 & 18.18 & 18.18 & 18.18 & 18.18 & 9.1 \\
\hline Composition of $\mathrm{CoCrCuFeNiSn}_{1}$, at.\% & 16.67 & 16.67 & 16.67 & 16.67 & 16.67 & 16.67 \\
\hline
\end{tabular}
\end{tabular}

The mass losses during ingot preparation did not exceed $1 \%$ and the average rate of cooling was $\sim 10^{2} \mathrm{~K} / \mathrm{s}$. The as-cast ingot was thereafter remelted and the nanostructured films were obtained from the melt by splat quenching (SQ) technique. A technique for splat quenching used in the present work consisted of rpid cooling of melt drops upon their collision with the internal surface of a rapidly rotating hollow cylinder of copper. The cooling rate was estimated following the methodology proposed in [13]. We used the expression (1)

$$
V=\frac{\alpha \vartheta}{c \rho \delta},
$$

where $c$ is the heat capacity of film, $\rho$ is the film density, $\alpha$ is the coefficient of heat transfer, $\vartheta$ is the excess temperature of the film and $\delta$ is the thickness of the film. Taking into account the thickness of fabricated splat quenched films, i.e., $\sim 50 \mu \mathrm{m}$, the estimated rate of cooling was $\sim 10^{6} \mathrm{~K} / \mathrm{s}$. The X-ray diffraction analysis (XRD) was carried out using a DRON-2.0 diffractometer with monochromatized $\mathrm{Cu} K \alpha$ radiation. The diffraction patterns were processed using QualX2 software [14]. The microhardness was examined using a tester PMT-3 at a load of $100 \mathrm{~g}$.

\section{Results and discussion \\ 3.1. Electronic, thermodynamic and atomic-size criteria of phase formation in high-entropy alloys}

There are two main criteria by which the high-entropy alloys are usually characterized. This is the entropy of mixing $\Delta S_{m i x}$ and the enthalpy of mixing $\Delta H_{m i x}$. However, to predict the phase composition of HEAs, some additional parameters were proposed $[1,15,16]$. These parameters include in particular the valence electron concentration (VEC), the thermodynamic parameter $\Omega$, which takes into account the melting temperature, mixing entropy and the mixing enthalpy. The important parameter is an atomic-size difference between alloy components which is denoted as $\delta$. Let's take a closer look at the above parameters

The basic principle of HEAs is the stabilization of the solid solution phase by the significantly higher configurational entropy of mixing $\Delta S_{\text {mix }}$ compared to conventional alloys. The configurational entropy of mixing during the formation of regular solution alloy can be determined as

$$
\Delta S_{m i x}=-R \sum_{i=1}^{n} c_{i} \ln c_{i},
$$

$c_{i}$ - atomic fraction of the $i$-th component, $R$ - universal gas constant. Increasing of mixing entropy reduces the Gibbs free energy of the alloy and improves the stability of 
the solid solution. For the alloy where $n$ is the number of components maximum mixing entropy is when they are mixed in equal atomic fractions.

Usually in HEAs $\Delta S_{m i x}$ value is in the range of $12-19 \mathrm{~J} /(\mathrm{mol} \cdot \mathrm{K})$. Due to the high mixing entropy HEAs are solid solutions typically having simple crystal structures (FCC or BCC), but to avoid the appearance of brittle intermetallic compounds, complex microstructures and amorphous phases in the structure of alloys, some phase formation criteria are required to be completed. According to $[1,15]$, the $\Omega$ parameter can be used to estimate the phase composition of HEA.

$$
\Omega=\frac{T_{m} \Delta S_{m i x}}{\left|\Delta H_{m i x}\right|}
$$

where $T_{m}$ is the average melting temperature of alloy and $\Delta H_{m i x}$ - mixing enthalpy

$$
\begin{gathered}
T_{m}=\sum_{i=1}^{n} c_{i}\left(T_{m}\right)_{i}, \\
\Delta H_{m i x}=\sum_{i=1, i \neq j}^{n} \Omega_{i j} c_{i} c_{j},
\end{gathered}
$$

where the regular melt-interaction parameter between $i$-th and $j$-th elements $\Omega_{i j}=4 \Delta H_{\text {mix }}^{A B}$, and $\Delta H_{\text {mix }}^{A B}$ - mixing enthalpy of binary liquid $\mathrm{AB}$ alloy. Alloy components should not have a large atomic-size difference, which is described by the parameter

$$
\delta=100 \sqrt{\sum_{i=1}^{n} c_{i}\left(1-r_{i} / \bar{r}\right)^{2}},
$$

where $\bar{r}=\sum_{i=1}^{n} c_{i} r_{i} ; r_{i}$ - the atomic radius of the $i$-th element.

According to [15] the HEA alloys for which $\Omega \geq 1.1$ and $\delta \leq 6.6 \%$ can form the solid solutions without intermetallic compounds and amorphous phases. However, simple (not ordered) solid solutions form if $-15 \mathrm{~kJ} / \mathrm{mol}<\Delta H_{m i x}<5 \mathrm{~kJ} / \mathrm{mol}$ and $\delta \leq 4.6 \%$.

The other useful parameter is the valence electron concentration, $V E C$, which has been proven useful in determining the phase stability of high entropy alloys [16]. VEC is defined by:

$$
V E C=\sum_{i=1}^{n} c_{i}(V E C)_{i},
$$

where $(V E C)_{i}$ - valence electron concentration (including the $d$-electrons) of the $i$-th element. As pointed in [16] at $V E C \geq 8.0$, the sole FCC phase exists in the alloy; at $6.87 \leq$ $V E C<8.0$, mixed FCC and BCC phases will co-exist and the sole BCC phase exists at $V E C<6.87$. At the same time, other relationships are given in [17]: the formation of solid solution with an FCC structure should be expected at VEC $>8.2$; solid solutions with a BCC structure are formed at $4.25<$ VEC $<7.2$; at $7.2<$ VEC $<8.2$, two-phase solid solutions with BCC and FCC crystal lattices are expected to be formed; and at VEC < 4.25 , an HCP crystal lattice is expected. 
Using the data from [18], we calculated $\Delta S_{m i x}, \Delta H_{m i x}, \delta$, VEC, $\Omega$ and $\gamma$ of the CoCrCuFeNiSn ${ }_{x}$ HEA (Tab. 1).

Electronic, thermodynamic and atomic-size parameters of the CoCrCuFeNiSn $\mathrm{x}$ high-entropy alloys

\begin{tabular}{|c|c|c|c|c|c|}
\hline Alloy & $\Delta S_{\text {mix }}, \mathrm{J} /(\mathrm{mol} \cdot \mathrm{K})$ & $\Delta H_{\text {mix }}, \mathrm{kJ} / \mathrm{mol}$ & $\Omega$ & VEC & $\delta, \%$ \\
\hline CoCrCuFeNiSn $_{0.5}$ & 14.69 & 4.23 & 5.72 & 8.36 & 7.08 \\
\hline CoCrCuFeNiSn $_{1.0}$ & 14.89 & 4.29 & 4.72 & 8.00 & 8.95 \\
\hline
\end{tabular}

\subsection{Structure and mechanical properties of $\mathrm{CoCrCuFeNiSn} n_{x}$ high-entropy alloys}

The phase composition of the investigated alloys, crystal lattice parameters, and the fine structure parameters (coherently scattering domain size and microstrains) (Tab.3) were determined from the XRD patterns (Fig. 1). The dislocation density $\rho$ was obtained from the profile of the first diffraction peak.

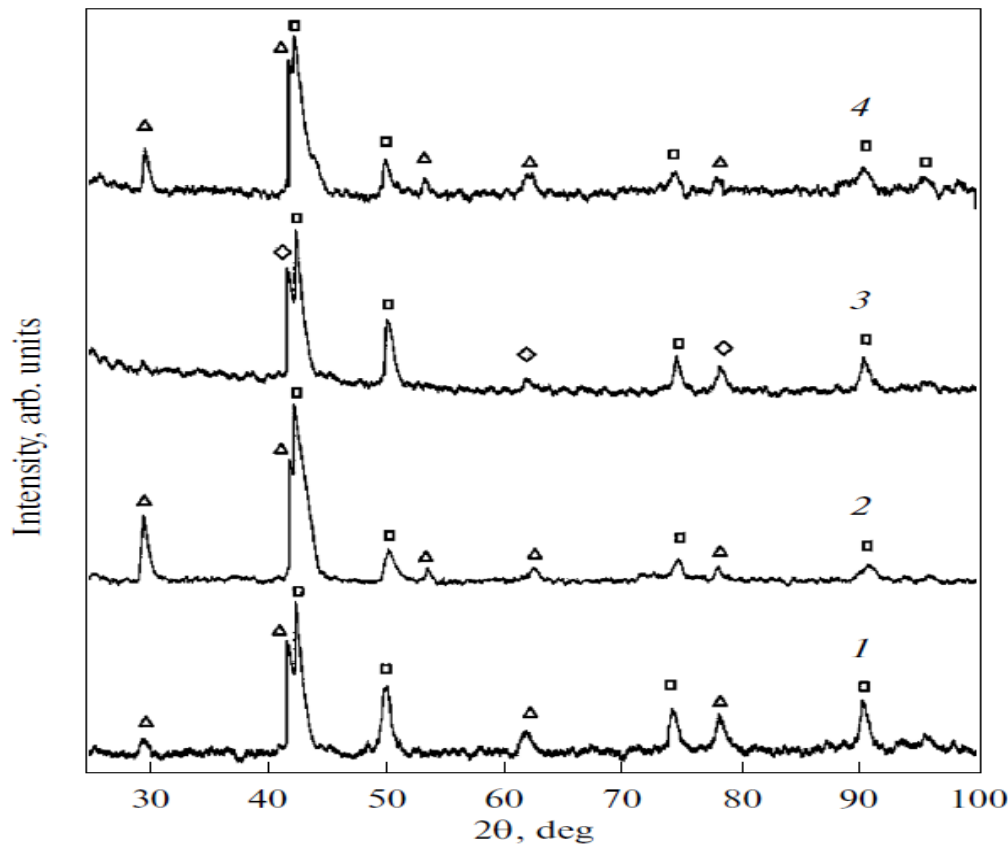

Fig. 1. XRD patterns of high_entropy alloys:( $\square)$ FCC; $(\Delta) B 2 ;(\diamond)$ BCC; (1) CoCrCuFeNiSn0.5, ascast; (2) CoCrCuFeNiSnı.o, as-cast; (3) CoCrCuFeNiSno.5, splat-quenched (SQ); (4) CoCrCuFeNiSn1.0, splat-quenched (SQ).

Table 3

The phase composition, coherently scattering domain size (crystallite size) $(L)$, the degree of distortion of the crystal lattice $(\Delta a / a)$, and the dislocation density $(\rho)$ of the investigated alloys

\begin{tabular}{|c|c|c|c|c|}
\hline Alloy & Phase composition & $L, \mathrm{~nm}$ & $\Delta a / a$ & $\rho, \mathrm{cm}^{-2}$ \\
\hline As-cast $\mathrm{CoCrCuFeNiSn} 0.5$ & $\begin{array}{l}\text { FCC }(a=0.3586 \mathrm{~nm})+\mathrm{BCC}(\mathrm{CsCl}- \\
\text { type, } a=0.2979 \mathrm{~nm})\end{array}$ & $\begin{array}{l}L_{\mathrm{FCC}}=37 \pm 2 \\
L_{\mathrm{BCC}}=27 \pm 2\end{array}$ & $1.8 \cdot 10^{-3}$ & $4.2 \cdot 10^{11}$ \\
\hline SQ film CoCrCuFeNiSno. & $\begin{array}{l}\text { FCC }(a=0.3588 \mathrm{~nm})+\text { BCC (CsCl- } \\
\text { type, } a=0.2974 \mathrm{~nm})\end{array}$ & $\begin{array}{l}L_{\mathrm{FCC}}=33 \pm 2 \\
L_{\mathrm{BCC}}=38 \pm 2\end{array}$ & $1.8 \cdot 10^{-3}$ & $5.2 \cdot 10^{11}$ \\
\hline As-cast $\mathrm{CoCrCuFeNiSn} 1.0$ & $\begin{array}{l}\text { FCC }(a=0.3600 \mathrm{~nm})+\text { BCC (CsCl- } \\
\text { type, } a=0.2981 \mathrm{~nm})\end{array}$ & $\begin{array}{l}L_{\mathrm{FCC}}=19 \pm 2 \\
L_{\mathrm{BCC}}=21 \pm 2\end{array}$ & $2.3 \cdot 10^{-3}$ & $1.6 \cdot 10^{12}$ \\
\hline SQ film CoCrCuFeNiSn ${ }_{1.0}$ & $\begin{array}{l}\text { FCC }(a=0.3600 \mathrm{~nm})+\mathrm{BCC}(\mathrm{CsCl}- \\
\text { type, } a=0.2987 \mathrm{~nm})\end{array}$ & $\begin{array}{l}L_{\mathrm{FCC}}=16 \pm 2 \\
L_{\mathrm{BCC}}=29 \pm 2\end{array}$ & $2.7 \cdot 10^{-3}$ & $2.5 \cdot 10^{12}$ \\
\hline
\end{tabular}

The analysis of the XRD patterns allowed us to establish the following: in both the as-cast and SQ samples, a two-phase (FCC + BCC) structure is formed. With increasing Sn content, the tendency of an ordered B2-type $(\mathrm{CsCl})$ phase to form increases in the 
BCC lattice of the alloy. This tendency becomes weaker with increasing rate of cooling to $10^{6} \mathrm{~K} / \mathrm{s}$. At the same time, in accordance with the criteria considered earlier, both of $\mathrm{CoCrCuFeNiSn}$ x HEA should have an FCC structure. These discrepancies between theoretical predictions and the actual phase composition are obviously explained by the fact that the theoretical criteria describe rather an idealized alloy. At the same time in a real alloy, the decomposition, precipitates and inhomogeneity of the chemical composition are observed [2]. It is obvious that, according to [17], we should assume that exact boundaries of the VEC range, which corresponds to the formation of two-phase solid solutions with BCC and FCC crystal lattices, are individual for each concrete alloy. It should be noted that the increased value of the parameter $\delta$ rather indicates the possibility of the existence of ordered solid solutions or intermetallic compounds, which is confirmed experimentally by the XRD patterns.

The XRD diffraction analysis made it possible to estimate the average density of dislocations in the cubic phases that arise in the alloy. It has been revealed that the SQ samples have a significantly greater (by about an order of magnitude) dislocation density compared to the as-cast alloys. This estimate of the dislocation density in the highentropy SQ alloys is comparable with the level of the dislocation density in heavily deformed samples. Splat quenching leads also to a change in the lattice parameters of the phases, which indicates the broadening of their ranges of homogeneity.

The SQ method ensures a noticeable decrease in the sizes of the coherently scattering domains, increases the level of microstresses, and substantially (by a factor of 1.6-2.0) increases the microhardness (to $8000 \mathrm{MPa}$ for the CoCrCuFeNiSn 1.0 alloy). As can be seen from Tab.4, an increase in the Sn content positively affects the microhardness, which is primarily due to an increase in the degree of elastic deformation of the crystal lattice caused by the introduction in its sites of large Sn atoms, as well as by the facilitation of the formation of the B2-type ordered phase in the alloy structure.

Microhardness of the CoCrCuFeNiSn
\begin{tabular}{|c|c|}
\hline Alloy & $H_{\mu}$, MPa \\
\hline As-cast CoCrCuFeNiSn 0.5 & $3500 \pm 200$ \\
\hline SQ film CoCrCuFeNiSn 0.5 & $5700 \pm 300$ \\
\hline As-cast CoCrCuFeNiSn 1.0 & $4000 \pm 200$ \\
\hline SQ film CoCrCuFeNiSn 1.0 & $8000 \pm 300$ \\
\hline
\end{tabular}

Table 4

\section{Conclusions}

1. In high-entropy $\mathrm{CoCrCuFeNiSn}$ alloys, an increase in the rate of cooling leads to the formation of supersaturated solid solutions based on bcc and fec lattices.

2. An increase in the concentration of $\mathrm{Sn}$ in the $\mathrm{CoCrCuFeNiSn}$ alloys favors the formation of an ordered (B2 type) solid solution in the structure of the alloys.

3. The positive influence of $\mathrm{Sn}$ on the increase in the microhardness revealed in the alloys studied appears to be due to not only an increase in the degree of distortions of the crystal lattice but also to the formation of an ordered B2-type phase. This conclusion is based on the fact that the alloy microhardness increases with an increasing degree of ordering in the solid solution.

\section{References}

1. Murty, B. S. High-entropy alloys. 2nd edition / B.S. Murty, J. W.Yeh, S. Ranganathan, P.P. Bhattacharjee. - Elsevier, 2019. - 363 p.

2. Bashev, V.F. Structure and properties of cast and splat - quenched high-entropy $\mathrm{Al}-\mathrm{Cu}-\mathrm{Fe}-\mathrm{Ni}-\mathrm{Si}$ alloys / V.F. Bashev, O.I. Kushnerov // Physics of Metals and Metallography. - 2017. - V. 118, No. 1. - P. 39-47. 
3. Miracle, D.B. A critical review of high entropy alloys and related concepts/ D.B. Miracle, O.N. Senkov // Acta Materialia. - 2017. - V. 122. - P. 448-511.

4. Wang, S. TiZrNbTaMo high-entropy alloy designed for orthopedic implants: ascast microstructure and mechanical properties/ S. Wang, J. Xu // Materials Science and Engineering: C. $-2017 .-$ V. 73. - P. 80-89.

5. Pogrebnjak, A.D. Irradiation resistance, microstructure and mechanical properties of nanostructured (TiZrHfVnBTa)N coatings/ A.D. Pogrebnjak, I.V. Yakushchenko, O.V. Bondar, V.M. Beresnev, K. Oyoshi, O.M. Ivasishin, H. Amekura, Y. Takeda, M. Opielak, C. Kozak // Journal of Alloys and Compounds. - 2016. - Vol. 679. - P. 155-163.

6. Rozman, K.A. Long-term creep behavior of a CoCrFeNiMn high-entropy alloy / K.A. Rozman, M. Detrois, T. Liu, M. C. Gao, P.D. Jablonski, J.A. Hawk // Journal of Materials Engineering and Performance. - 2020. - Vol. 29, No. 9. - P. 5822-5839.

7. Li, Y. New soft magnetic $\mathrm{Fe}_{25} \mathrm{Co}_{25} \mathrm{Ni}_{25}(\mathrm{P}, \mathrm{C}, \mathrm{B})_{25}$ high entropy bulk metallic glasses with large supercooled liquid region/ Y. Li, W. Zhang, T. Qi // Journal of Alloys and Compounds. - 2017. - Vol. 693. - P. 25-31.

8. Xu, Y. Formation and properties of Fe25Co25Ni25(P, C, B, Si)25 high-entropy bulk metallic glasses / Y. Xu, Y. Li, Z. Zhu, W. Zhang // Journal of Non-Crystalline Solids. - 2018. - V. 487.- P. 60-64.

9. Ding, J. High entropy effect on structure and properties of $(\mathrm{Fe}, \mathrm{Co}, \mathrm{Ni}, \mathrm{Cr})-\mathrm{B}$ amorphous alloys/ J. Ding, A. Inoue, Y. Han, F. L. Kong, S. L. Zhu, Z. Wang, E. Shalaan, F. Al-Marzouki // Journal of Alloys and Compounds. - 2017. - V. 696. - P. 345-352.

10. Dudnik, E.F. Higher order ferroic properties of TGS monocrystals / E.F. Dudnik, A.I. Kushnerev, V.M. Duda //Materials Research Innovations. -1999. -Vol.2, No.5. -P.309-311.

11. Dudnik, E.F. Second-order ferroic properties of a Pb5Ge3O11 uniaxial ferroelectric / E.F. Dudnik, V.M. Duda, A.I. Kushnerov // Physics of the Solid State. 2001. - Vol. 43, No. 12. - P. 2280-2283.

12. Kushnerov, O.I. Structure and properties of nanostructured metallic glass of the $\mathrm{Fe}-\mathrm{B}-\mathrm{Co}-\mathrm{Nb}-\mathrm{Ni}-\mathrm{Si}$ high-entropy alloy system / O.I. Kushnerov, V.F. Bashev, S.I. Ryabtsev // Springer Proceedings in Physics, -2021. - P. 557-567.

13. Miroshnichenko, I.S. Quenching from the Liquid State/ I.S. Miroshnichenko. Metallurgiya, Moscow, 1982.-168p. [in Russian].

14. Altomare, A. Main features of Qualx 2.0 software for qualitative phase analysis / A. Altomare, N. Corriero, C. Cuocci, A. Falcicchio, A. Moliterni, R. Rizzi // Powder Diffraction. - 2017. - Vol. 32, No. S1. - P. S129-S134.

15. Guo, S. Phase stability in high entropy alloys: Formation of solid-solution phase or amorphous phase /S. Guo, C.T.Liu// Progress in Natural Science: Materials International.-2011.-V. 21, Iss. 6, -P. 433-446.

16. Guo, S. Effect of valence electron concentration on stability of fcc or bcc phase in high entropy alloys /S. Guo, C. Ng, J.Lu, C.T.Liu// Journal of Applied Physics.-2011.V. 109, Iss. 10, -P. 103505-1-103505-5.

17. Krapivka N.A. Features of phase and structure formation in high-entropy alloys of the $\mathrm{AlCrFeCoNiCu} x$ system $(\mathrm{x}=0,0.5,1.0,2.0,3.0)$ / N.A. Krapivka, S.A. Firstov, M. V Karpets, A. N. Myslivchenko, V. F. Gorban' // The Physics of Metals and Metallography. - 2015. - Vol. 116, No. 5. - P. 467-474.

18. Takeuchi, A. Classification of bulk metallic glasses by atomic size difference, heat of mixing and period of constituent elements and its application to characterization of the main alloying element/A. Takeuchi, A. Inoue// Materials Transactions.-2005. -V. 46-P. 2817-2829. 